\title{
Pipe-line SM-devices for power application
}

\author{
R.R. Ionaytis, A.Yu. Vasilenko, V.F. Lisovoy and M.A. Tooktarov \\ Research and Development Institute of Power Engineering, P.O. Box 788, 101000 Moscow, \\ Russia
}

\begin{abstract}
Nuclear power plants (NPP) are supplied with numerous and various pipeline devices (PLD) that are in hard operating conditions. The number of PLD failures at NPP have already reached $35 \%$ of total number of component failures. The main directions of PLD modernization based on shape memory (SM) devices are considered in this paper. Quick replaceable flat-annular fixing-sealing SM-elements for valve seat sealing and welded joint protection are proposed. Small-size non-reducing electrically-driven progressive SM-actuators, passive (directacting) SM-initiators of valves at accident changes of power plant parameters (temperature, pressure, low water level, neutron flux, etc.) are discussed.
\end{abstract}

\section{INTRODUCTION}

The capabilities of SM allows and the scope of SM devices (SMD) application in nuclear power were believed to be boundless at first. Facilities for research and development were established and pilot implementation was started. Successful results were obtained. It was found that various SMD for nuclear power might be developed $/ 1-2 \%$.

Since the conditions of SM alloys and SMD operation at the nuclear installations are very difficult, the concept of their application at NPP was developed /3/. The capabilities and scope of application were identified, in particular, for mounting and repair, for influence on reactivity, for shut off pipelines $13-5 /$. Speeding up implementation of SM alloys and SMD for engineering purposes was discussed in detail in the report $/ 6 /$, patents in review $/ 5,7-8 /$.

Now protective flat-annular fixing-sealing elements are proposed /9-11/. Actuators that can be actively (by electrical drive) controlled, in general, based on a single SMA crystals /12-14/ and passive initiating devices for actuation have been developed /9-10/.

\section{PROBLEMS OF PLD. PURPOSES AND MEANS OF THEIR SOLUTION}

PLDs are designed, manufactured, tested and operated in compliance with strict Regulations and Standards in Nuclear Power. However they account for up to $25 \%$ of all failures at the power unit and up to $40 \%$ of its downtime. $70 \%$ of repair costs come for PLDs. PLDs are designed to meet entrenched engineering solutions (in some cases adopted several dozens years ago), therefore they may fail to comply with up-to-date reliability and safety practices.

Usually required capability of PLDs are currently complemented with the following requirements: reduced mass and dimensions, easy-to-fix speed of response, passive actuation, vibration and seismic resistance, adaptability for diagnostics, well-developed servicing, controllability of service life (its possible extension to 60 years).

\section{UNCONVENTIONAL MEANS OF THE PROBLEMS PLD SOLUTION}

The above-specified purposes and capabilities of PLDs can be attained in the following way:

- the use of reliable engineering solutions proven in operation;

- designing the valves with a view to meet the whole set of reliability and safety criteria: 
internal safety; well-equipped; support to implementation of assigned functions; structural division; consistency in function implementation; automatism; provision of fast response; safe failure; structural, functional, time, information redundancy; independence (in terms of functions and positions); diversity; defense-in-depth; passive actuation; protection from external impact (men-made or produced by loose items); controllability; testability in standard operating conditions; single-purpose design; low energy rating; modular design; simplicity of design solutions and maintenance; harmless operation (non-injurity of operation); user-friendliness (for the operating personnel); completion of action, once started;

- the use of unconventional means;

including such as:

- application of valves designed with different principles of action and/or design- engineering realization and intended for implementation of the same function (fast opening or closure);

- passive protection (actuated automatically on power operation) of pipeline welded joints, first of all (in places where valves are joined) by means of flat-annular fixing SM elements that would significantly extend the service life of welded joints and simplify valve arrangement (thanks to reduction of valve number, their location in part or totally outside the containment);

- development and implementation of easy-to-replace removable parts, installation of non-expendable fixing and sealing SM elements;

- designing of emergency devices operating in accordance with the control logic principle "2 out of 3";

- provision of easy transformation of PLDs actuators to different forces and travel distance by means of piston-spring actuators to be used in safety systems (piston drive provides activation and holding, springs work for actuation);

- preference for vibration- and seismic-resistant plate-spring actuators for the use in a wide range of PLDs travel distances, including gates designed for travel within hundreds of millimeters;

- the use of passive initiators of actuation (PIA): in response to loss of power (low voltage), change in rate or direction of coolant flow, low level of coolant in the core, high pressure and/or temperature in the compartments, rise of neutron flux;

- development of midget (powerful) SM-actuators for PLDs to be installed at small-size pipelines.

\section{FLAT-ANNULAR FIXING-SEALING ELEMENTS}

Fixing-sealing SM-elements are proposed, first of all, for reinforcement of pipeline welds which are typically in tension under the impact of inner fluid, can not work in tension, which is why small (nonthrough) cracks are developed in 15 to 20 years.

To protect welds in a passive way by means of reduction of tensile stresses, it is proposed to put SM strip clamps in martensitic state (Fig. 1) on the pipeline without interference from one or both sides of the weld (i.e. power unit shutdown is not required). The clamps come with fasteners which do not protrude off the outer diameter of the clamp, while the external clamps may have protruding fasteners $/ 9-10 /$.

When a pipeline is heated (NPP is brought to power), SM clamps go to austenitic state with compression strain of $6-8 \%$ from the initial clamp length. This produces tensile stress. In case of cooling (for example, NPP is shut down for repair) clamp material goes to martensitic state (that decreases stress 3 to 8 times) and does not produce pressure onto the pipeline.

Even for thick-wall pipes stress in welded joints can be reduced almost to zero with designeracceptable width and thickness of SM clamps. The number of SM clamps depends on technological capabilities and expediency. 

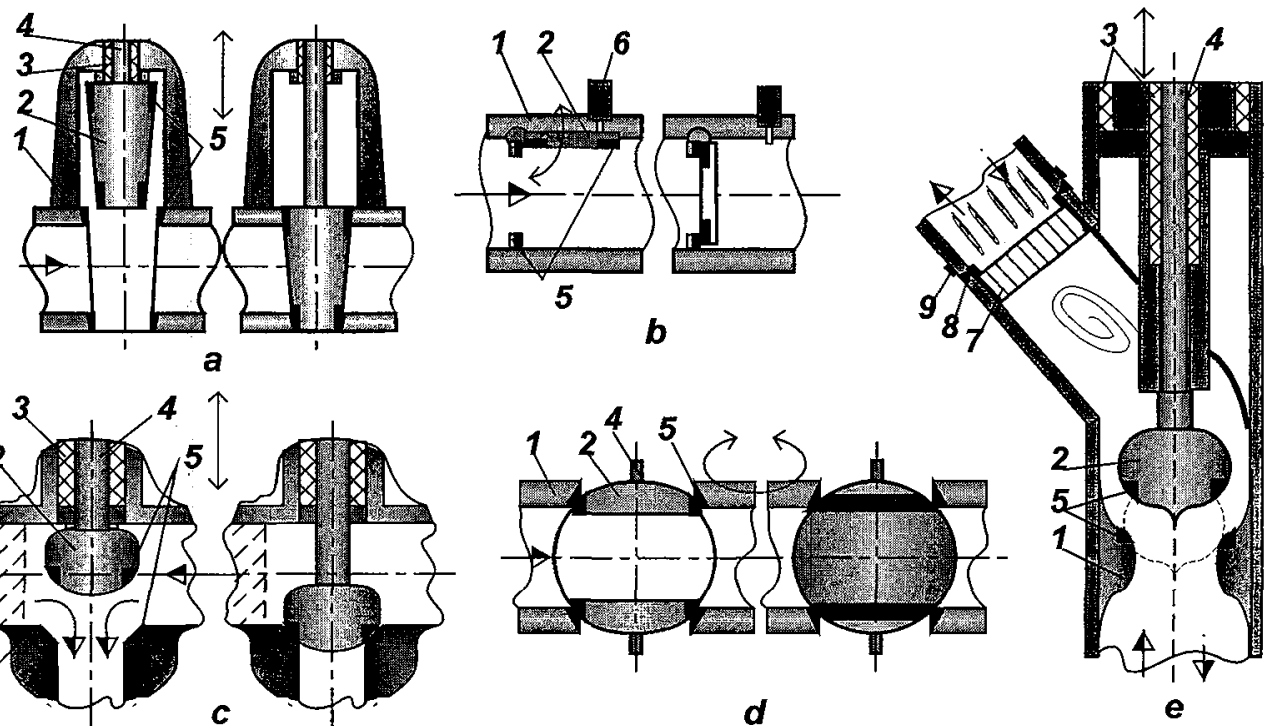

Fig. 1. Flowing part: a-gate valve; b-check slide globe valve (with position indication); c-angular valve (dashed line) or twowoy valve (cutout-throttle, safety); d-valve cock; e-valve (cutoff) with downward nozzle and vortex rectifier; 1-body; 2-slide valve; 3-packing seal; 4-rod; 5-seat sealing; 6-position indicator; 7-vortex rectifier; 8-weld; 9-clamp

Another efficient application of SM fixing-sealing elements is quick replacement of removable parts of valve seats and gates for installation of wear-resistant seat-plunger pairs (see Fig. 1). Such high resistance to wear results from very high SMD resistance to erosion - in 5-6 times higher than for other knows surfacing. Such SMD application will facilitate PLD servicing and reduce personnel occupational doses.

\section{DIRECT ACTING INITIATING DEVICES}

Most PLDs are controllable by the operator's command. PLDs qualified as safety devices (SD) are usually controllable automatically by the instrumentation commands. Such PLDs can be initiated following the control logic principle " 2 out of 3 ". They are equipped with three detectors arranged in such a way that at least two signals will cause initiation.

Some PLDs are direct acting devices, i.e. they respond to direct impact of fluid. These devices are simple in design but they initiate only by the control logic principle " 1 out of 1 ", i.e. in response to only one scram signal (which may be spurious signal).

To improve reliability of SD actuation in nuclear power, it is strongly recommended to use direct acting devices implementing the principle of passive operation, in other words, they are initiated by an emergency event (without the operator's command) irrespective of the operation of any active means (control device or power source). Therefore, it is required to complement the design of direct acting PLD (such as check and safety valves, etc.) with passive devices that can automatically come into action under various accidents (loss of power supply, ruptures of pipelines, pressure changes, etc.).

To control pipe-line devices (PLD) at NPP, diverse means are available (Fig. 2):

- active PLDs (gates and valves) are initiated by control safety system following CSS-T technology; 
- passive PLDs are initiated by their own passive initiators;

- direct acting devices (check gate valves, safety valves, rupture disks) are controlled directly by working fluid.

The signals from flow rate, pressure, temperature, power, etc. detectors come through three trains, as a minimum, as required by Regulations and Standards in Nuclear Power. The signals are processed by the control logic principle " 2 out of 3 " and are directed to electrically-geared actuators of active valves.

The signals from each passive initiator (by pressure, temperature, level, flow rate) go through three trains to control units where they are caused initiation of three-way electromagnetopneumatic switches following the control principle " 2 out of 3" (no electronic devices are used), switch off pneumatic drives and switch on spring drives.

The passive initiator of actuation responded to neutron flux (and reactor power) increase was tested at a distance of 340-390 mm above the core in IVV-2M reactor in October, 1990. Its working body - springs made of $\mathrm{Mn}-\mathrm{Cu}$ alloy - was subjected to long-time irradiation at $75 \mathrm{oC}$, the electric contacts were closed at $80.5 \pm 2.5^{\circ} \mathrm{C}$, and open - at $83.5 \pm 4^{\circ} \mathrm{C}$. The ratio of neutron fluxes found in 8 experiments is $1.25 \pm 1.7 \mathrm{oC}$.

To make possible power-free control, three-support holding-starting SM device sensitive to neutron flux increase has been developed. In response to neutron flux increase this PIA will activate the casing with a conical protrusion, flat SM elements are fixed on support tube of the working body by means of a fastening ring.

The number of holding SM elements is sufficient for realization of the control logic principle "2 out of $3 "$. It was proven by experiments that PIA-F could hold the working body with $50 \mathrm{~N}$ force at $20^{\circ} \mathrm{C}$ temperature. It actuates almost instantaneously as soon as the threshold temperature of SMA $\left(\approx 100^{\circ} \mathrm{C}\right)$ is exceeded.

A passive initiator of actuation in response to temperature rise in the compartment (PIA-T) has been developed on the basis of bent plates made of SM alloy (titanium nickelide). Once straightened, the plates come in contact with a microswitch (opening the electrical contacts) if the preset temperature is reached. Reduction of fluid temperature and, consequently, temperature of SM alloy plate returns it to the initial state under the effect of the microswitch spring. As it takes place, the contacts close again. Welldetermined temperature of SM alloy phase transition permits to rely on reproducibility of initiator's actuation.

A passive initiator of actuation in response to coolant level decrease in the reactor vessel (PIA-H) has been designed taking thermoelectrical level transducer as a prototype. PIA-H indicates changes in temperature at liquid-steam boundary owing to a difference in heat transfer coefficients for liquid and steam phases. PIA-H will demonstrate long-term performance at high temperature $(\approx 280 \mathrm{oC})$ and pressure $(\approx 7 \mathrm{MPa})$ without any significant change in its parameters under the effects of irradiation. In case of accident level decrease in the reactor vessel (by $0.3 \mathrm{~m}$ ) PIA-H will de-energize, the contacts come open that initiates actuators (following the control logic principle "2 out of 3").

Developments are being underway for PIA controlling flow rate on the basis of temperature change corresponding with the respective feedwater temperature $(\approx 190 \mathrm{oC})$, and with accident temperature $(\approx 2800 \mathrm{oC})$ of subcooling water at reactor outlet.

\section{ELECTRICALLY DRIVEN SM ACTUATORS}

Available electrically driven valve actuators are designed and manufactured in compliance to longstanding (for dozens of years) technical solutions. They have relatively large mass and dimensions. Therefore thousands (sometimes dozens of thousands) of valves installed at small-size pipelines ( DN larger than $6 \mathrm{~mm}$ ) are controlled manually. 


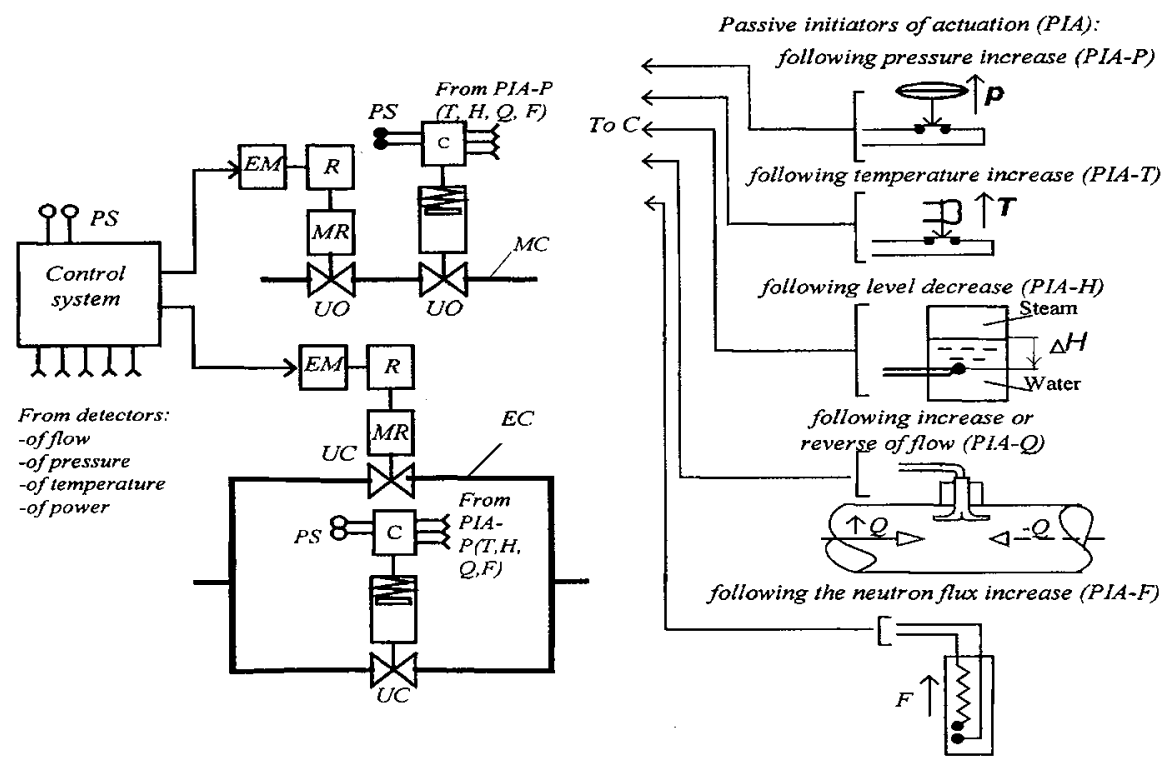

Fig. 2. A schematic diagram of PLD control: UO-usually open; UC-usually closed; $C$-controller; $F$ - neutron flux; $Q$-fluid flow rate; P-pressure; T-temperature; $H$ - level; EM-electric motor; R-reducer; MR-moving reformer; PS-power supply, PIApassive initiator of actuation; $M C$-main circuit; $E C$-emergency circuit

For nuclear power plants of the next century it seems reasonable to enter new capabilities to the market, viz. remote electrical control of small-size valves at pulse pipelines (I\&C and others). For this purpose it is necessary to develop and implement small-size no-gear electrically driven forward motion and revolving actuators based on SM rods. They are intended primarily for small-size valves (DN larger than $6 \mathrm{~mm}$ ) and ball valve cock.

Such SM actuators with opposite placement of SM closing and opening drives are being designed and tested in RDIPE (Fig. 3).

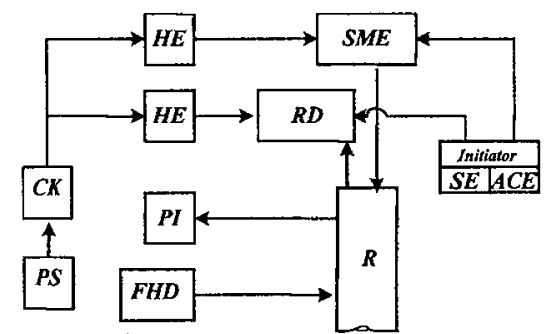

Fig. 3. A schematic diagram of thermo-response SM valve. $R D$ - return device; $P I$ - position indicator; $C K$ - control key (switch); HE- heating element; SMA-SM actuator; $A C E$ amplifying-converting element; FHD. fixing and holding device; $S E$ - sensor element; $R$ - rod of working body; $P S$ power supply

From 4 to 12 actuators in the form of rod and tubular single crystals of $\mathrm{Cu}-\mathrm{Al}-\mathrm{Ni}$ are used per one drive. A travel distance of $2.5-5 \mathrm{~mm}$ forces amount from 1.5 to $3.5 \mathrm{t}$ if the mass of SM actuator varies from 0.6 to $1.6 \mathrm{k}$ Control is effected by means of heating elements ranged in power from 0.1 to $0.9 \mathrm{~kW}$ in the range of actuatio temperature from 80 to $200^{\circ} \mathrm{C}$, speed of actuation is $10-30 \mathrm{~s}$ under pipe internal pressure $0 \ldots 7 \mathrm{MPa} / 12 /$. 


\section{CONCLUSIONS}

Pipeline devices which are very important for the safety of nuclear power plants shall be designed to achieve a higher level of reliability and safety of their operation as well as space-saving capability and maintainability. This objective can be attained by the following means: complementing of direct acting devices with passive features that come into action from direct acting initiators; diversity of operation of safety devices; the use of passive fixing-sealing elements; designing of actuation initiators (upon loss of power, low coolant level in the reactor, temperature or/and pressure change) using the control logic principle " 2 out of 3 ".

The above specified PLDs with SM capabilities would allow to reach high parameters and performance.

\section{REFERENCES}

1. L.Mc.D.Schetky. The Present Status of Industrial Applications for SMA's, in Proceedings of SMA's for Power Systems (L. McD. Schetky, eds.), Palo-Alto, Nov 1994, EPRI-TR-105072, Project 9000, p.4.1.

2. Ionaitis R.R., et al. SMA in The Russian Nuclear Power Industry, p. 10.1 (see [1]).

3. Ionaitis R.R. Concept of Functional Materials Use in Unconventional Means For Nuclear Plants, in Proceedings of The Third International Conference on Intelligent Materials (P.F. Gobin, J.Tatibouet, eds.), June 1996, France, Lyon, SPEE, V. 2779, p. 974.

4. Ionaitis R.R. Possibilities and Peculiarities of SMA Use in Power Engineering, in SMST-97: Proceedings of the Second International Conference on Shape Memory and Superelastic Technologies (A.Pelton, et al, eds.), Pacific Grove, California, USA, March 1997, p.974.

5. Ionaitis R.R., Tooktarov M.A. Patent Investigation of SMA Application in NPP, Atomnaja tecknika za rubegom (Nuclear Engineering International), №2, 1997, p.3.

6. Ionaitis R.R., Chernov D.B. On Possibilities to Accelerate Engineering of Shape Memory Alloys, in Proceedings of the ESOMAT-97, Netherlands, Enschede, 1997, p. 643.

7. Ionaitis R.R., Tooktarov M.A. Patent Investigation of SM Actuators, Atomnaja tecknika za rubegom (Nuclear Engineering International), №4, 1999, p.12.

8. Ionaitis R.R., Tooktarov M.A. Patent Investigation of Thermosensitive Valves, Atomnaja tecknika za rubegom (Nuclear Engineering International), №9, 1999, p.3.

9. Ionaitis R.R. Directly Acting Pipe-Line SM Devices for Nuclear Power Plants, in Proceedings of the SMM'99, Kanazawa, Japan, May 1999, p.51.

10. Abramov V.Ya, Perspectives of Creation of the Protective System with SM-Elements for Nuclear Reactors, in Proceedings of the International Symposium «Gateway to the $21^{\text {st }}$ Century», Quebec, Canada, Aug. 1999, p.449.

11. Vasilenko A.Yu. Physical fundamentals of application of single crystal with shape memory, in Proceedings of the Conference «Deformation and failure mechanisms of advanced materials». Pskov, Russia, Sept.1999, p.353.

12. Ionaitis R.R. Up-grading of pipe-line devices by unconventional means // Atomnaya energiya (Nuclear energy). 1998. V.84. Is.4. p.363-356.

13. Ionaitis R.R., Sinitzin Eu.N., Tooktarov M.A., Vlasova E.M. Possibilities of passive protection of weld joints based on flat-annular fixing-sealing SM-elements, in Proceedings of "Pipe-line safety", MoscowPushkino. 1999. V.2, p.214-222.

14. Ionaitis R.R., et al. SM-Actuator of Power Valve, in Proceedings of the SMST, Amsterdam, 1999. 